Full paper

\title{
Two-dimensional metal phosphorus trisulfide nanosheet with solar hydrogen-evolving activity
}

\author{
Fengmei Wang ${ }^{\mathrm{a}}$, Tofik Ahmed Shifa ${ }^{\mathrm{a}, \mathrm{b}}$, Peng $\mathrm{He}^{\mathrm{c}}$, Zhongzhou Cheng ${ }^{\mathrm{d}}$, Junwei Chu ${ }^{\mathrm{a}}$, Yang Liu ${ }^{\mathrm{a}, \mathrm{b}}$, \\ Zhenxing Wang ${ }^{\mathrm{a}}$, Feng Wang ${ }^{\mathrm{a}}$, Yao Wen ${ }^{\mathrm{a}}$, Lirong Liang ${ }^{\mathrm{e}}$, Jun $\mathrm{He}^{\mathrm{a}, \mathrm{b}, *}$ \\ a CAS Center for Excellence in Nanoscience, CAS Key Laboratory of Nanosystem and Hierarchical Fabrication, National Center for Nanoscience and Technology, Beijing \\ 100190, PR China \\ b University of Chinese Academy of Sciences, Beijing 100049, PR China \\ c State Key Laboratory of Chemical Resource Engineering, Beijing University of Chemical Technology, Beijing 100029, PR China \\ d School of Materials Science and Engineering, University of Science and Technology Beijing, 100083, PR China \\ e Center for Physicochemical Analysis and Measurement, Institute of Chemistry, Chinese Academy of Sciences, Beijing 100190, PR China
}

\section{A R T I C L E I N F O}

\section{Keywords:}

Two dimensional

Few layered

Metal phosphorus trisulfide

Chemical vapor deposition

Water splitting

\begin{abstract}
A B S T R A C T
The development and utilization of photocatalysts to realize water-splitting without any external bias or sacrificial agents has received the limelight. As a novel two-dimensional layered material, metal phosphorus trichalcogenides (MPTs) cause wide research interest, presently. However, the growth of ultrathin two-dimensional MPT crystals is a great challenge to hinder their application. Here, we initially grow few-atomic layered nickel phosphorus trisulfide $\left(\mathrm{NiPS}_{3}\right)$ as promising photocatalyst for hydrogen evolution. The as-prepared NiPS 3 hexagonal nanosheet, as thin as few atomic layers $(\leq 3.5 \mathrm{~nm}$ ), has lateral size of larger than $15 \mu \mathrm{m}$. These ultrathin $\mathrm{NiPS}_{3}$ crystals can directly generate hydrogen gas from pure water without any sacrificial agents under sunlight. With ultraviolet photoelectron spectrometer and electrochemical impedance spectroscopy, we show that the attractive photocatalytic activity of the ultrathin $\mathrm{NiPS}_{3}$ crystals arise from their appropriate positions of the band edges. This discovery is expected to make a contribution to develop next generation solar-fuel conversion catalysts for $\mathrm{H}_{2}$ production.
\end{abstract}

\section{Introduction}

Generating hydrogen from water by utilizing solar energy is a potentially scalable approach to meet energy demand without any environmental crisis $[1,2]$. To date, various water-splitting methods are established for using the gigantic energy from the sun. A complicated photovoltaic electrolysis (PV) system, where the solar cell powers an electrolyzer, has been regarded as one of the efforts developed to realize the splitting of water. In some cases, additional supply of electrical power is required to assist the photocatalysis, namely photoelectrocatalysis, to derive the up-hill water splitting reaction. Compared with the above two methods, photocatalysis under mere irradiation of sunlight for water splitting is a much more facile approach [3]. But, in this system, the plentiful sacrificial agents usually have to be added in water to consume the photogenerated holes and boost the generation of hydrogen gas. Therefore, the solar-driven overall water splitting without the use of external bias or sacrificial agents is of particular interest due to its simplicity and low cost. During the past decades, some single semiconductor based photocatalysts, such as nanocrystalline $\mathrm{CoO}$ [4] and $\left(\mathrm{Ga}_{1-\mathrm{x}} \mathrm{Zn}_{\mathrm{x}}\right)\left(\mathrm{N}_{1-\mathrm{x}} \mathrm{O}_{\mathrm{x}}\right)$ solid solutions [5] were investigated for $\mathrm{H}_{2}$ generation through water-splitting.

Recently, apart from these metal oxides, layered two-dimensional (2D) materials have received significant attention due to their unique chemical and physical features [6-9]. The 2D nature provides the possibility to reduce the electron-hole recombination via shortening the migration distance and increase the activity of interfacial reactions [10-12]. In this regard, the $2 \mathrm{D}$ structured $\mathrm{g}-\mathrm{C}_{3} \mathrm{~N}_{4}$ [13-16] has been proved as a promising photocatalyst under simulated solar light irradiation. Yet, its photoactivity for $\mathrm{H}_{2}$ evolution is evaluated in the solution containing the sacrificial electron donor $[13,17]$ or in the presence of co-catalysts $[15,18,19]$. So, the development of novel 2D nanomaterials capable of producing $\mathrm{H}_{2}$ gas from pure water is highly desirable. Notably, metal phosphorus trichalcogenides (MPTs), a new kind of 2D semiconductors, are proposed [20,21] and theoretically studied [22] for water splitting nowadays. Their wide bandgaps from $1.3 \mathrm{eV}$ to $3.5 \mathrm{eV}$ endow them good photoelectronic properties and

\footnotetext{
* Corresponding author at: CAS Center for Excellence in Nanoscience, CAS Key Laboratory of Nanosystem and Hierarchical Fabrication, National Center for Nanoscience and Technology, Beijing 100190, PR China.

E-mail address: hej@nanoctr.cn (J. He).
} 
a

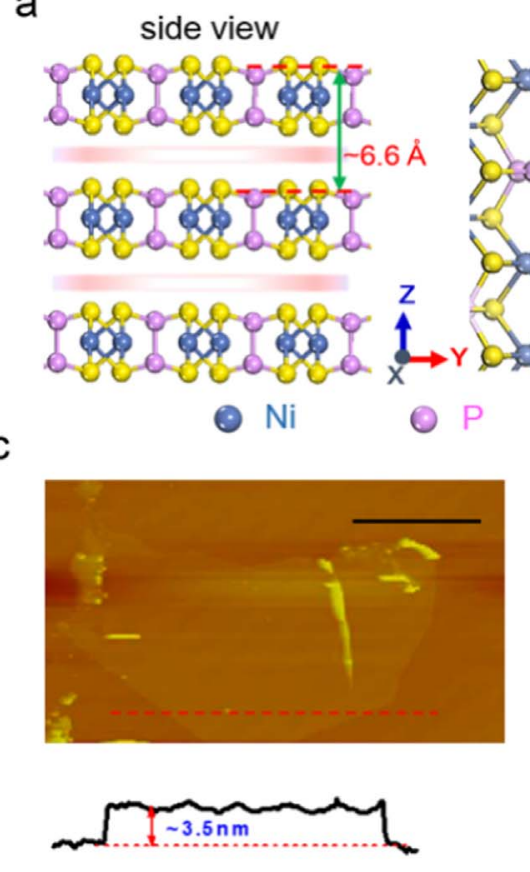

b
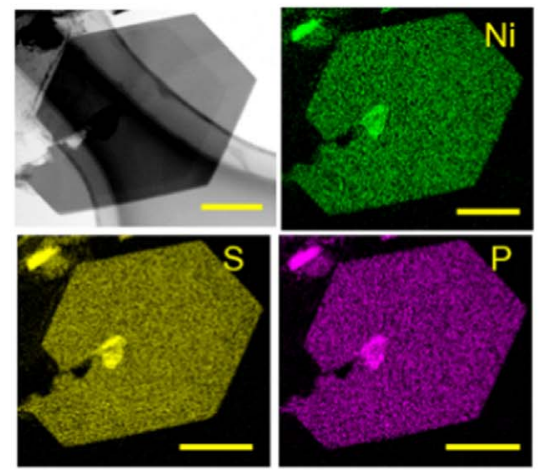

d

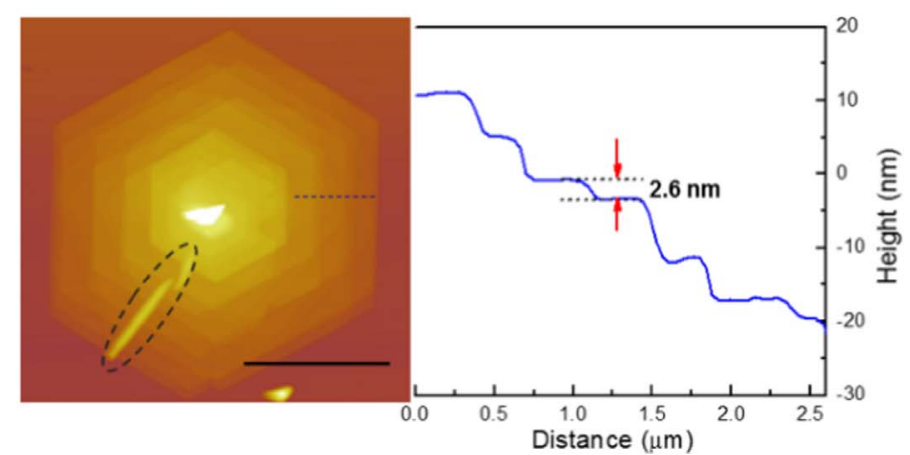

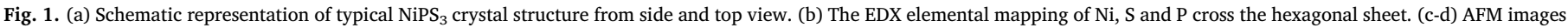
and the corresponding height analysis of the hexagonal few layered and multilayered NiPS ${ }_{3}$ crystals. Scale bar, (b) $500 \mathrm{~nm}$ and (c-d) $5 \mu \mathrm{m}$.

photocatalytic activities. And their strong absorption in visible-light region and high carrier mobility $\left(625.9 \mathrm{~cm}^{2} \mathrm{~V}^{-1} \mathrm{~s}^{-1}\right.$ for $\left.\mathrm{MnPSe}_{3}\right)[22]$ suggest the potential ability for $\mathrm{H}_{2}$ production under visible light. Unfortunately, only the bulk crystals of MPTs have so far been synthesized via the chemical vapor transport (CVT) process [21,23-25]. The method employs the heating of stoichiometric amount of high-purity elemental metal, phosphorus and sulfur powders in the evacuated quartz tube at high temperature $\left(>600{ }^{\circ} \mathrm{C}\right)$ for couple of days. As to the nanostructured MPTs, it has remained a great challenge to successfully make the synthesis and hence there has been no report about the nanoscale growth of such materials hitherto.

Here, we develop a chemical vapor deposition (CVD) process with the mixed sulfur and phosphorus powder as well as various substrates grown $\mathrm{Ni}(\mathrm{OH})_{2}$ nanosheets to initially grow ultrathin 2D hexagonal nickel phosphorus trisulfide $\left(\mathrm{NiPS}_{3}\right.$ ) crystals in large area. The resulting $\mathrm{NiPS}_{3}$ nanosheet can be as thin as a few atomic layers, with the lateral sizes up to a dozen of micrometers $(>10 \mu \mathrm{m})$ and high crystalline quality. This crystal comprises covalently bonded $\left(\mathrm{P}_{2} \mathrm{~S}_{6}\right)^{4-}$ bipyramids, located in the center of six nearby $\mathrm{Ni}^{2+}$, and the layers stack in $\mathrm{C} 2 / \mathrm{m}$ space group via van der Waals force (Fig. 1a). One single-layer has the thickness around $0.66 \mathrm{~nm}$. Significantly, these $\mathrm{NiPS}_{3}$ nanosheets can decompose pure water to produce hydrogen gas under Xe light $(\lambda>300 \mathrm{~nm})$ and simulated solar light without any co-catalysts or sacrificial agents. The $\mathrm{H}_{2}$ evolution rate under Xe light and simulated solar light is about 26.42 and $6.46 \mu \mathrm{mol} / \mathrm{g} / \mathrm{h}$, respectively. Using ultraviolet photoelectron spectrometer and electrochemical impedance spectroscopy, we show that the attractive photocatalytic activity of the 2D ultrathin $\mathrm{NiPS}_{3}$ crystals arise from their appropriate positions of the band edges. To the best of our knowledge, this work is the first to communicate the facile synthesis of ultrathin $\mathrm{NiPS}_{3}$ crystals and demonstrate its photocatalytic activity.

\section{Experimental section}

\subsection{CVD growth of $2 D$ ultrathin $\mathrm{NiPS}_{3}$ crystals}

Firstly, the ultrathin $\mathrm{Ni}(\mathrm{OH})_{2}$ nanosheets were prepared on the carbon fibers (CFs), fluorine doped tin oxide (FTO) and indium tin oxide (ITO) glasses with the size of $2 \times 4 \mathrm{~cm}^{2}$ through the typical hydrothermal method (Supporting information) [26]. The tube furnace with two zones was used in the growth (Fig. S1). The CFs or FTO glasses grown $\mathrm{Ni}(\mathrm{OH})_{2}$ nanosheets were then put in a quartz tube to act as the growth substrates, located in the right zone (downstream) of the furnace, for the growth of $2 \mathrm{D}$ ultrathin NiPS 3 crystals (Fig. S1). Appropriate amount $(1.0 \mathrm{~g})$ of highly purified phosphorous (red phosphorus, $98.5 \%)$ and sulfur powders (99.5\%) with the molar ratio of $n_{\mathrm{p}}: n_{\mathrm{S}}=2: 5$ were grinded together and kept on the left zone (upstream) in another quartz tube. The distance between right zone and left zone is $\sim 26 \mathrm{~cm}$. Subsequently, the right and left zones were simultaneously heated to $470{ }^{\circ} \mathrm{C}$ and $\sim 270{ }^{\circ} \mathrm{C}$ within $30 \mathrm{~min}$ under $\mathrm{Ar}$ gas (20 sccm, 99.999\%), respectively. The reaction lasted to $60 \mathrm{~min}$ at $470{ }^{\circ} \mathrm{C}$. To ensure the completed reaction, the mixture of $\mathrm{P}$ and $\mathrm{S}$ powder was finally heated to $300{ }^{\circ} \mathrm{C}$. Before each growth, oxygen was carefully removed through a repeated purging (with Ar gas, 99.999\%) and pumping process until the pressure down to $0.1 \mathrm{~Pa}$. After the growth, the samples were cooled to room temperature and protected in a small serum bottle filled with argon gas (99.999\%) for further measurements.

\subsection{Structural characterization}

The morphology of the ultrathin $\mathrm{NiPS}_{3}$ crystals was identified by scanning electron microscopy (SEM, Hitachi S4800), the thickness by atomic force microscopy (AFM, MFP-3D Infinity), and the chemical composition, crystalline quality and detailed crystal structure by transmission electron microscopy (TEM, FEI Tecnai F20, $200 \mathrm{kV}$ for selected area electron diffraction (SAED) measurement and HRTEM observations; JEM-2100F, $200 \mathrm{kV}$ for STEM- energy dispersive X-ray spectroscopy (EDX) elemental mapping and EDX spectra). Atomic-level 
HAADF-STEM images were obtained from a $300 \mathrm{kV}$ STEM (JEM ARM200F). X-ray diffraction (XRD, D/MAX-TTRIII (CBO) diffractometer) using $\mathrm{Cu} K \alpha$ radiation $(\lambda=1.5418 \AA$ ) was used to check the crystal structure. Raman scattering was performed on a confocal microscope-based Raman spectrometer (Renishaw InVia) in ambient air environment with an excitation laser line of $532 \mathrm{~nm}$. X-ray photoemission spectra (XPS) were recorded on ESCALAB $250 \mathrm{Xi}$ system of Thermo Scientific, where the analysis chamber was $1.5 \times 10^{-9} \mathrm{Mbar}$ and the X-ray spot was $500 \mu \mathrm{m}$. The valence band energy of the NiPS crystals were analyzed on Thermo Scientific ESCALab 250Xi using ultraviolet photoelectron spectroscopy (UPS). The gas discharge lamp was used for UPS, with helium gas admitted and the HeI $(21.22 \mathrm{eV})$ emission line employed. The helium pressure in the analysis chamber during analysis was about $2 \mathrm{E}^{-8}$ mbar. The data were acquired with $-10 \mathrm{~V}$ bias. Room temperature UV-Vis diffuse reflectance spectra was recorded using a Lambda 750 spectrophotometer in the wavelength range of $400-1000 \mathrm{~nm}$.

\subsection{Electrochemical impedance measurement}

In order to measure the band edge positions (or flatband potentials) of ultrathin $\mathrm{NiPS}_{3}$ crystals, a piece of carbon fibers (CFs) grown $\mathrm{NiPS}_{3}$ acted as working electrode in typical three-electrode system with the platinum wire as a counter electrode and SCE as a reference electrode. The measured area of the working electrode was $\sim 1 \mathrm{~cm}^{2}$. The electrolyte was $0.1 \mathrm{M} \mathrm{Na}_{2} \mathrm{SO}_{4}$ solution $(\mathrm{pH}=6.59)$. Electrochemical impedance spectroscopy (EIS) measurements were carried out using a Princeton Applied Research (PAR) Potentiostat/ Galvanostat VersaSTAT3. Both the potentiostat and the lock-in amplifier were controlled by a computer using VersaStudio software. The potential was scanned between 0.0 and $2.1 \mathrm{~V}$ with various modulation frequencies. All the potentials reported here are relative to RHE. Potential conversion between SCE and RHE is given by: $\mathrm{E}(\nu s \mathrm{RHE})=\mathrm{E}(v s \mathrm{SCE})+0$. $242 \mathrm{~V}+0.059 \times \mathrm{pH}$.

\subsection{Photocatalytic activity test}

The photocatalytic reactions of the photocatalysts were carried out in an outer irradiation-type photoreactor (Pyrex glass) connected to a closed gas-circulation system. The $\mathrm{NiPS}_{3}$ nanosheets covered carbon fibers with the size of $\sim 8 \mathrm{~cm}^{2}$ (the mass loading of $\mathrm{NiPS}_{3}$ crystals about $1.25-1.88 \mathrm{mg} / \mathrm{cm}^{2}$ ) were directly immersed in $100 \mathrm{~mL}$ ultrapure water (Millipore, $18.2 \Omega$ ). A $300 \mathrm{~W}$ Xe-lamp and a cutoff filter was applied to achieve AM 1.5G solar simulator. The photocatalytic $\mathrm{H}_{2}$ evolution rate was analyzed using an on-line GC-7900 gas chromatograph (GC,TCD detector, 5A molecular sieve column and Ar carrier). Magnetic stirrer was used during the water splitting experiment to ensure the gas release. The catalyst was used for continuous water splitting up to one day.

\subsection{Theoretical calculations}

The spin-polarized calculations on the bulk and slab $\mathrm{NiPS}_{3}$ were both performed by adopting the Vienna ab-initio simulations package (VASP) code [27]. The interaction between ions and electron was described by the project augmented wave (PAW) [28]. And the generalized gradient approximation (GGA) expressed by the functional of Perdew, Burke and Ernzernhof (PBE) [29]. A cutoff energy was set to $500 \mathrm{eV}$ for structure optimization and electronic structure calculation. The single-layer $\mathrm{NiPS}_{3}$ was cleaved from the (001) crystal face of bulk $\mathrm{NiPS}_{3}$ and modeled by a $2 \times 2$ supercell. Thus an $8 \% \mathrm{~S}$ vacancy concentration (close to the experiment value 7\%) can be obtained by removing two $S$ atoms. A Monkhorst-Pack mesh of $9 \times 9 \times 1$ was used for slab model and $6 \times 3 \times 5$ for the bulk. In addition, a vacuum space with $15 \AA$ was inserted between the adjacent $\mathrm{NiPS}_{3}$ sheets. During geometry optimization, all the atoms were fully relaxed until the residual forces were less than $0.02 \mathrm{eV} / \AA$.

\section{Results and discussion}

We synthesized 2D ultrathin $\mathrm{NiPS}_{3}$ crystals with a high density via simple chemical vapor deposition (CVD) method (Fig. S1) starting from the $\mathrm{Ni}(\mathrm{OH})_{2}$ nanosheets (Fig. S2) [26], elemental sulfur and phosphorous precursors. The relatively mild reaction condition at $450-470{ }^{\circ} \mathrm{C}$ enables the growth of $\mathrm{NiPS}_{3}$ nanosheets on various substrate, including carbon fibers (CFs), fluorine doped tin oxide (FTO) and indium tin oxide (ITO) glasses (Fig. S3). At the beginning, small irregular nanosheets with the size below $500 \mathrm{~nm}$ were formed (Fig. S4a). A longer growth time leaded to the increase in the size and the change in morphology of the nanosheets (Fig. S4b-c). At a lower growth temperature $\left(\leq 400{ }^{\circ} \mathrm{C}\right)$, the reaction would be not finished and the nanosheet had relatively smaller size along with lots of residual reactant (Fig. S5a). But, when the growth temperature was brought above $500{ }^{\circ} \mathrm{C}$, the $\mathrm{NiPS}_{3}$ nanosheets would be converted to the "squamae" (Fig. S5c-f) due to the etching process at high temperature [30]. Therefore, we used the growth temperature of $450-470{ }^{\circ} \mathrm{C}$ to fabricate hexagonal NiPS ${ }_{3}$ crystals (Fig. S4c and Fig. S5b) within 60 min. After CVD growth, the ultrathin $2 \mathrm{D} \mathrm{NiPS}$ crystals can be transferred to $\mathrm{SiO}_{2}$ / $\mathrm{Si}$ substrate or TEM grids for detailed structural characterization and measurements. Fig. 1b shows a low- magnification annular dark-field TEM image of a hexagonal 2D crystal. The EDX and corresponding EDX mapping indicates the crystal is composed of homogeneous Ni, P and $\mathrm{S}$ distribution with the atomic ratio of $\sim$ 1:1:3 (Fig. S6).

The ultrathin $\mathrm{NiPS}_{3}$ nanosheet, transferred onto $\mathrm{SiO}_{2} / \mathrm{Si}$ substrate, has the thickness of $\sim 3.5 \mathrm{~nm}$ and lateral size of $\sim 15 \mu \mathrm{m}$ as can be seen from AFM image in Fig. 1c. Interestingly, AFM image (Fig. 1d) of some complete $\mathrm{NiPS}_{3}$ hexagon clearly exhibits the spiral structure topology because of the introduction of a "pike" from the residue of precursor at initial stage (Fig. S7) [31-33]. It consists of multilayered hexagons with the thickness of each layer down to $\sim 2.6 \mathrm{~nm}$. The structure of $\mathrm{NiPS}_{3}$ crystals was further studied with XRD pattern. The sharp peaks in Fig. 2a can be attributed to the monoclinic $\mathrm{NiPS}_{3}$ (PDF No.33-0952). The Raman spectrum, excited by $532 \mathrm{~nm}$ laser light, of the $\mathrm{NiPS}_{3}$ nanosheets on CFs is investigated in Fig. 2b. The first-order modes, including four in-plane $\mathrm{E}_{\mathrm{g}}\left(\sim 130, \sim 175, \sim 281\right.$ and $\left.\sim 555 \mathrm{~cm}^{-1}\right)$ and two out-of-plane $A_{1 g}\left(\sim 381\right.$ and $\left.\sim 585 \mathrm{~cm}^{-1}\right)$ phonon modes, dominate the spectrum $[25,34]$. Fig. 2c shows the XPS spectra (Fig. S8) of $\mathrm{NiPS}_{3}$ nanosheets in Ni2p, P2p and S2p regions. The binding energy of $\mathrm{Ni}_{2} \mathrm{p}_{3 / 2}(\sim 854.54 \mathrm{eV})$ and the present shake-up satellites features further confirm the $\mathrm{NiPS}_{3}$ chemical nature [35]. The prominent P2p peaks at $131.98 \mathrm{eV}\left(2 \mathrm{p}_{3 / 2}\right)$ and $132.80 \mathrm{eV}\left(2 \mathrm{p}_{1 / 2}\right)$ are assigned to covalent P-S. The S2 $\mathrm{p}_{3 / 2}$ and $\mathrm{S} 2 \mathrm{p}_{1 / 2}$ peaks, located at $\sim 162.50 \mathrm{eV}$ and $\sim 163.62 \mathrm{eV}$, respectively, are attributed to Ni-S and P-S [35,36]. It is of particular note that there is a slightly red shift in the binding energy for $\mathrm{P}_{2} \mathrm{p}_{3 / 2}$ and $\mathrm{S} 2 \mathrm{p}_{3 / 2}$ peaks compared to those $(\sim 132.32$ and $\sim 163.1 \mathrm{eV})$ of bulk crystal [35], which is resulted from the presence of sulfur vacancy in the sample. More about this will be discussed later.

The crystallographic structure and quality of $\mathrm{NiPS}_{3}$ nanosheet was further characterized by high-resolution TEM (HRTEM), selected-area electron diffraction (SAED) and scanning transmission electron microscopy (STEM). Fig. 3a clearly demonstrates the hexagonal morphology of the ultrathin $2 \mathrm{D} \mathrm{NiPS}$ crystal with the lateral size of $\sim 8 \mu \mathrm{m}$. The SAED pattern (Fig. 3b) along the [001] zone axis indicates the high crystallinity of the nanosheet. Additionally, a series of planes and relevant angles marked in the SAED pattern further reveal the monoclinic crystal system (PDF No.33-0952). Moreover, HRTEM image of the $\mathrm{NiPS}_{3}$ ultrathin crystal shows a honeycomb arrangement of the atoms with the lattice spacing of $0.267 \mathrm{~nm}$ and $0.252 \mathrm{~nm}$, corresponding to the (022) and (040) planes (Fig. 3c). The direct observation of the atoms on the basal plane is achieved through typical atomic-level highangle annular dark-field (HAADF)-STEM image owing to the contrast obtained by the presence and the nature of the atoms under the electron 

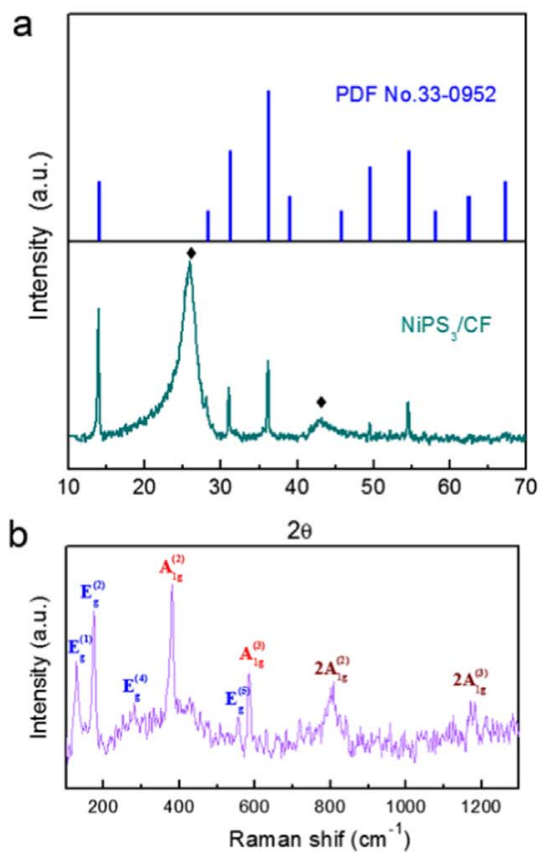
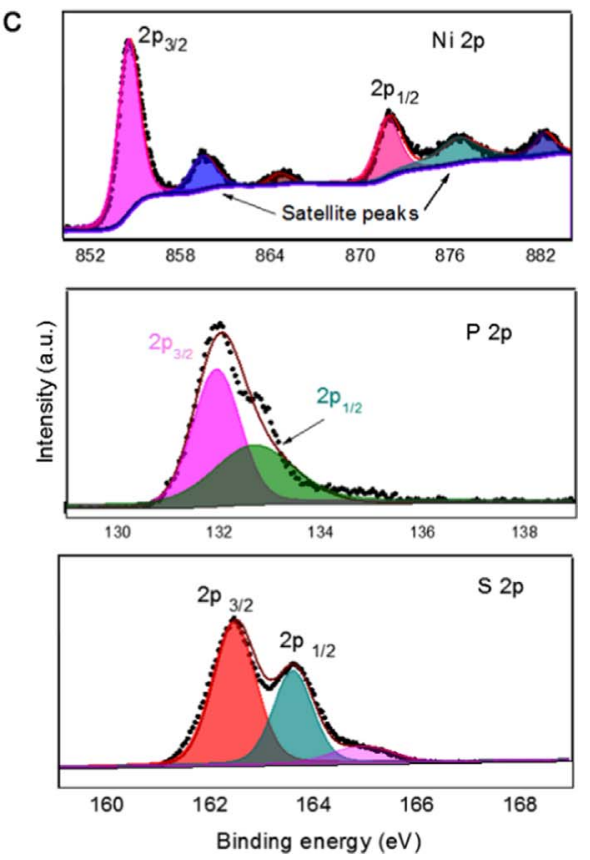

Fig. 2. (a-b) XRD pattern (" $"$ stands for the XRD pattern from $\mathrm{CFs}$ ) and Raman spectrum of $\mathrm{NiPS}_{3}$ on the CFs. (c) XPS analysis in Ni 2p, P 2p and S 2p regions, respectively. beam. As shown in Fig. 3d, inside the basal plane (001) per $\left[\mathrm{P}_{2} \mathrm{~S}_{6}\right]$ unit is found in the center of nearby six Ni atoms, exhibited by brightest spots. The $\mathrm{S}$ and $\mathrm{P}$ atoms can't be distinguished clearly because their molar mass is very close to each other. The atom arrangement is consistent with the crystal structure in Fig. 1a. Of particular note is that some $\mathrm{S}$ vacancies $\left(\mathrm{S}_{\mathrm{v}}\right)$ are observed in the $\mathrm{NiPS}_{3}$ nanosheet (Fig. 3e-f), similar with the S vacancies observation in $\mathrm{MoS}_{2}$ nanosheet [37-39]. Careful analysis of the $\mathrm{NiPS}_{3}$ basal plane reveals that the $\mathrm{S}$ vacancies is estimated as $\sim 7.6 \%$ (Fig. S9). This phenomenon is also consistent with the XPS analysis and gives rise to the ferromagnetism features (Fig. S10, S11 and Note SII).

Water-splitting experiments were performed in an air-tight flask with 2D ultrathin $\mathrm{NiPS}_{3}$ crystals on CFs in neutral pure water under
Xenon light or simulated AM1.5G solar illumination (Fig. 4a). The sign of generated $\mathrm{H}_{2}$ was quantified by gas chromatography (GC) trace using argon as the carrier gas (inset of Fig. 4a). And there is no quantifiable oxygen gas in this photocatalytic system (the detail discussion is in Note SI). As displayed in Fig. 4b, the generation of $\mathrm{H}_{2}$ is observed when the system is kept under the Xenon light $(\lambda>300 \mathrm{~nm})$. The $\mathrm{H}_{2}$ evolution proceeds continuously with the constant rate of $\sim 26.42 \mu \mathrm{mol} / \mathrm{g} / \mathrm{h}$ until turning off the light irradiation. As comparison, control experiment unveils that almost no $\mathrm{O}_{2}$ is detected when $\mathrm{AgNO}_{3}$ used as the sacrificial hole donor under the same condition (Supporting information). Nevertheless, when the $\mathrm{Na}_{2} \mathrm{~S} / \mathrm{Na}_{2} \mathrm{SO}_{3}$ as sacrificial electron donor, $\mathrm{H}_{2}$ could be produced with the rate of $74.67 \mu \mathrm{mol} / \mathrm{g} / \mathrm{h}$ (Supporting information, Fig. S12). These results suggest the $\mathrm{NiPS}_{3}$ nanosheets are not a
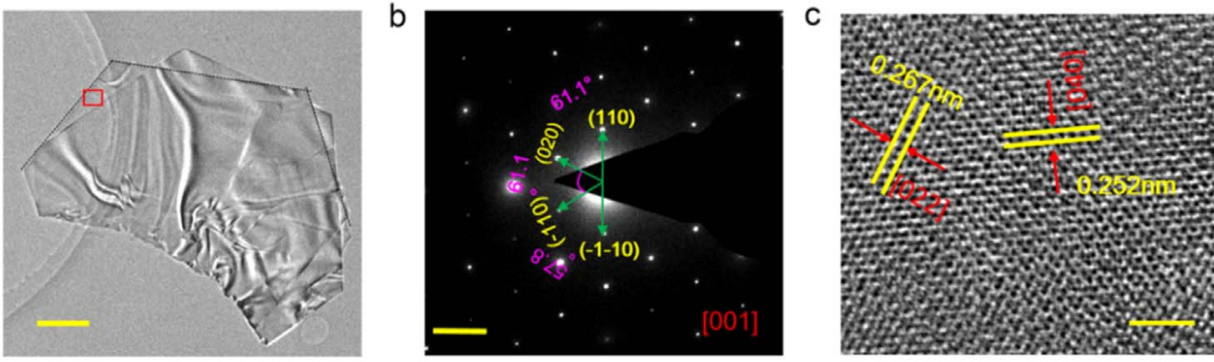

d

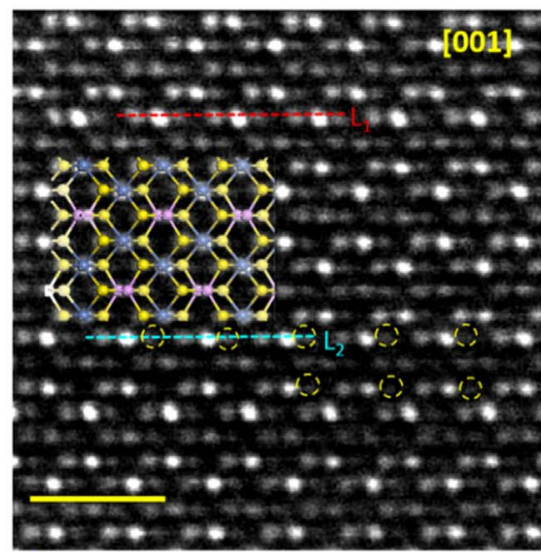

e

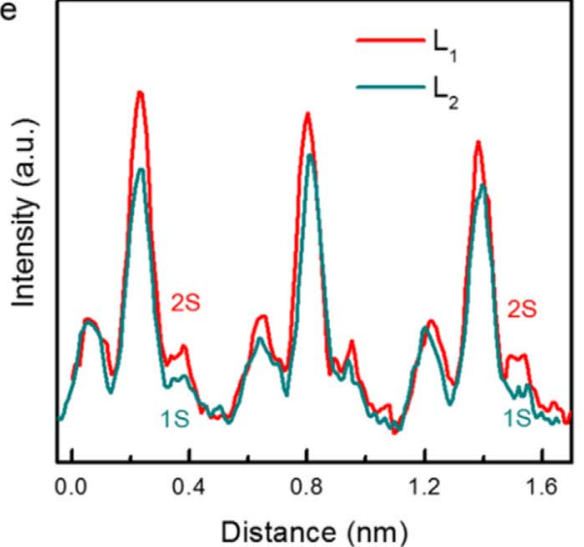

Fig. 3. (a-c) Low-magnification TEM image, SAED pattern along the [001] zone axis and HRTEM image marked in a of a hexagonal $2 \mathrm{D} \mathrm{NiPS}_{3}$ crystal. (d) Atomic-level HAADF-STEM image of an ultrathin $\mathrm{NiPS}_{3}$ nanosheet showing the sulfur vacancies (yellow circles) and the corresponding structural schematic. FFT mask filter has been employed for clarity. (e) Intensity profiles along lines L1-L2. Higher contract is obtained from the Ni atom compared to $\mathrm{S}$ atom. In absence of $\mathrm{S}$ atoms in this $\mathrm{NiPS}_{3}$ nanosheet (L2), the intensity decreases to $<50 \%$ compare with the intensity in L1. Scale bar, (a) $1 \mu \mathrm{m}$, (b) $21 / \mathrm{nm}$, (c) $2 \mathrm{~nm}$ and (d) $1 \mathrm{~nm}$. 

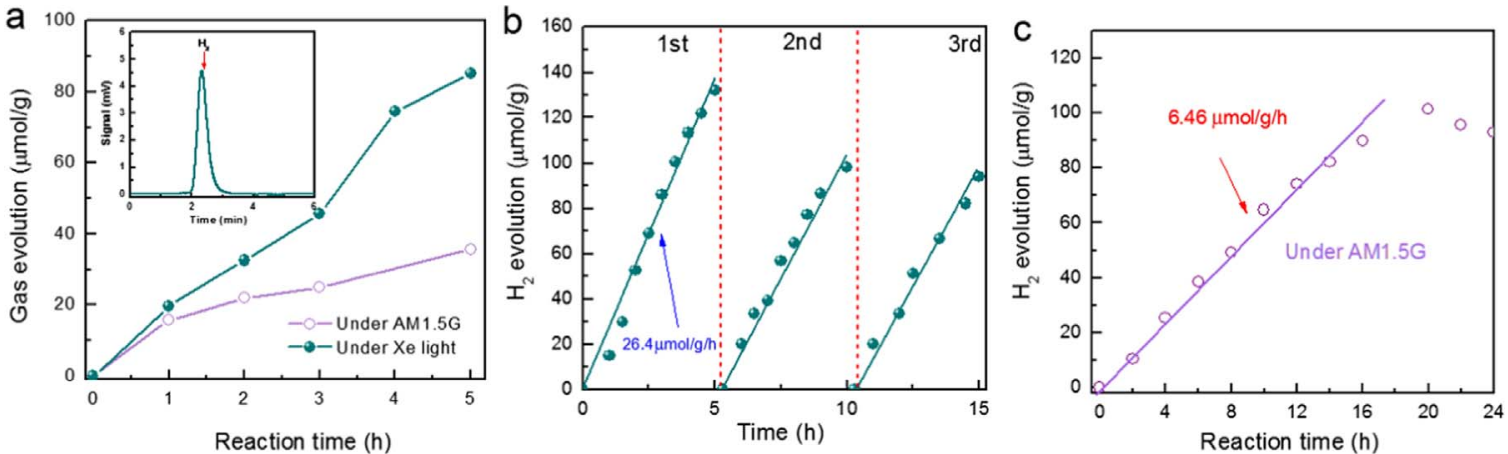

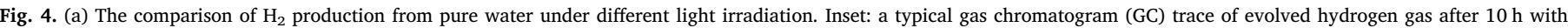
irradiation of Xe-Light. (b-c) Time course of $\mathrm{H}_{2}$ production from pure water under Xe light and AM 1.5G simulator within different irradiation time.

suitable for oxygen evolution under the irradiation of Xenon light. With the aim of extending the scenario towards more realistic phenomena, the photocatalytic experiment was conducted under illumination of simulated solar light and significant generation of $\mathrm{H}_{2}$ gas is observed in this condition too (Fig. 4c). The constant $\mathrm{H}_{2}$ evolution rate is $~$ $6.46 \mu \mathrm{mol} / \mathrm{g} / \mathrm{h}$. The superior stability, which turns out to be stable in continuous 20-h test, further indicates the promising practical potential (Fig. S13 and S14). These results corroborate the photocatalytic ability of the $2 \mathrm{D} \mathrm{NiPS}_{3}$ crystals to generate $\mathrm{H}_{2}$ gas from pure water without any sacrificial agent.

To understand the catalytic activity of the 2D NiPS 3 crystals and explore the reason why the stoichiometric oxygen gas could not be released in this system, we further studied the band structure [4]. Fig. 5a shows the reflectivity spectrum of the sample. The corresponding optical bandgap can be estimated from the modified Kubelka-
Munk function $\left((a h v)^{\mathrm{r}}\right.$ vs $h v$, the inset of Fig. 5a). A good linear fit when using $r=1 / 2$ is in accord with the previously calculated work [22] claiming $\mathrm{NiPS}_{3}$ to be an indirect bandgap material. The bandgap $\left(\mathrm{E}_{\mathrm{g}}\right)$ is close to $1.96 \mathrm{eV}$, a value similar to that reported in the literature $[40,41]$. To obtain absolute band-edge positions with respect to water redox potentials, we utilize the $\mathrm{NiPS}_{3}$ on CFs as an electrode to measure its flat-band potentials [42-44]. From the Mott-Schottky plots (Fig. 5b), the flat-band potential of $0.81 \mathrm{~V}$, the difference between Fermi level and water-reduction potential, is observed. Negative slopes of the MottSchottky plots indicate that the NiPS 3 is a $p$-type semiconductor. A similar result was observed elsewhere [45]. Here, we assumed that Fermi level is about $0.3 \mathrm{eV}$ above the valence band for $p$-type semiconductors $[43,46]$. The valence band position is thus estimated at $1.11 \mathrm{~V}$ (vs RHE, $\left.-5.55 \mathrm{eV} v s \mathrm{E}_{\text {Vacuum }}\right)$. Furthermore, ultraviolet photoelectron spectroscopy (UPS) was also used to determine the valence band energy level
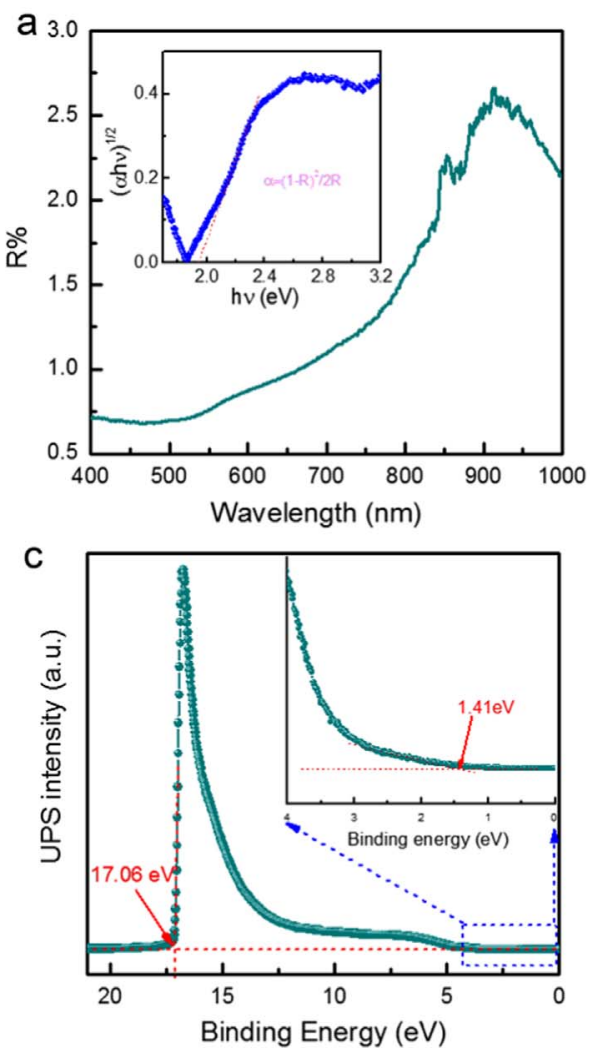

d
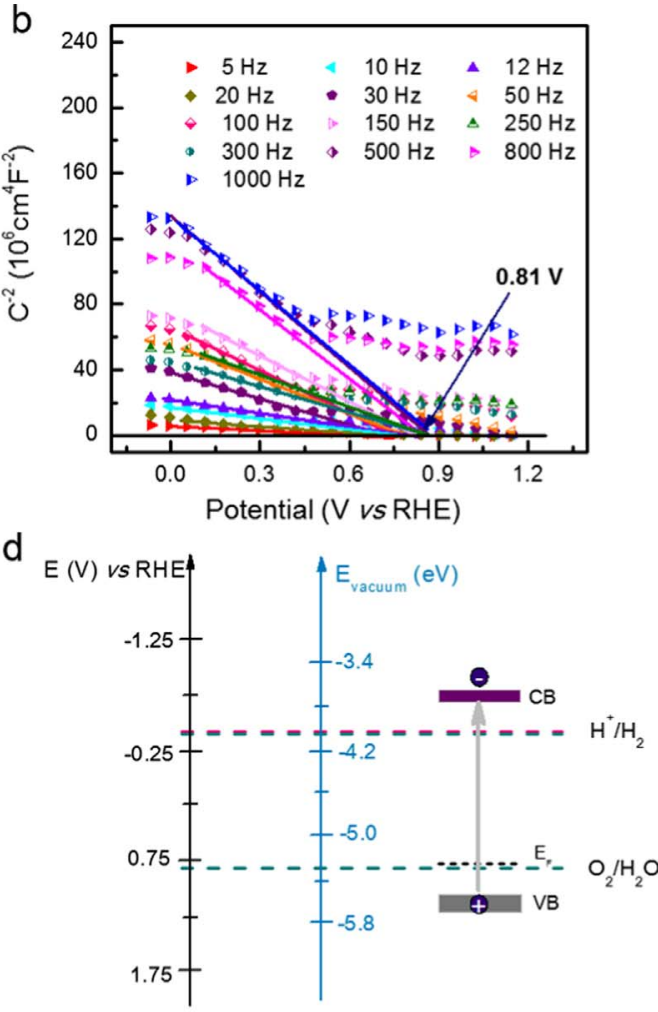

Fig. 5. (a) Ultraviolet-visible diffuse reflectance spectrum and the corresponding $(a h v)^{1 / 2}$ as a function of photon energy $(h v)$, where $a$ is the KubelkaMunk function of the diffuse reflectance $R$. The intercept of extrapolated straight line give the bandgap of $\mathrm{NiPS}_{3}$ crystals. (b) Mott-Schottky plots for $\mathrm{NiPS}_{3}$ nanosheets according to impedance measurements. The flat-band potential $(0.81 \mathrm{~V} v s$ RHE) is achieved from the intercept of the extrapolated lines. (c) UPS spectra of $\mathrm{NiPS}_{3}$ crystals, in which the dashed red lines mark the baseline and the tangents of the curve. The intersections of the tangents with the baseline give the edges of the UPS spectra based on the UPS width. (d) Band structure diagram for $\mathrm{NiPS}_{3}$ nanosheets according to the bandgap, flat-band potential and UPS edges obtained from (a-c). VB, valence band; $\mathrm{CB}$, conduction band. 
of $\mathrm{NiPS}_{3}$ crystals. The value is calculated to be $-5.57 \mathrm{eV}$ ( $v s \mathrm{E}_{\mathrm{Vacuum}}$ ), in agreement with the value from the M-S plot, by subtracting the width of He I UPS spectra (Fig. 5c) from the excitation energy (21.22 eV). The conduction band energy $\left(E_{c}\right)$ is then calculated as $\sim-3.61 \mathrm{eV}$ from $E_{\mathrm{v}^{-}}$ $E_{g}$. A more obvious illustration is given in Fig. $5 d$ comprising of the above discussed band edge energy positions. As can be seen, the conduction band of the ultrathin $\mathrm{NiPS}_{3}$ crystals is situated above the water reduction potential which is a thermodynamically feasible level for generating $\mathrm{H}_{2}$ gas. In so far the valence band is concerned, it is slightly below the oxidation level of $\mathrm{H}_{2} \mathrm{O}$ to $\mathrm{O}_{2}(-5.28 \mathrm{eV})$. Here comes the reason why there is no evolution of $\mathrm{O}_{2}$ gas in our photocatalytic system. A weak intrinsic driving force in band alignment of ultrathin 2D NiPS crystals with water oxidation potential is not energetically sufficient to supply the required holes for the generation of oxygen gas. As with many single-component photocatalysts, ultrathin $\mathrm{NiPS}_{3}$ nanosheets likewise suffer from the issue of stability-their activity reduces by half after about a one-day of reaction (Fig. 4b and d). The morphology and crystallinity of the $2 \mathrm{D}$ ultrathin $\mathrm{NiPS}_{3}$ crystals still retained after a long time reaction (Fig. S11), suggesting their stability is still better than that of nanocrystalline $\mathrm{CoO}$ photocatalyst [4]. The negligible change in the XPS spectra in Ni 2p region suggests the stability of $\mathrm{NiPS}_{3}$ nanosheets during the long-time reaction (Fig. S14b). While, the XPS spectra in P $2 p$ and $S 2 p$ regions demonstrate shifts of $\sim 0.34 \mathrm{eV}$ (Fig. S14c-d) to higher binding energy, which is due to the strong oxygen adsorption on the $\mathrm{P}$ and $\mathrm{S}$ atoms. And the XPS spectra in $\mathrm{O} 1$ s region indicate that the surface of the NiPS 3 is slightly oxidized after photocatalytic test (Fig. S15). As a matter of concept, DFT calculations about absorption energy of $\mathrm{O}$ atoms on the surface of $\mathrm{NiPS}_{3}$ (Fig. S16) corroborates the observed surface oxidation. There exists the strong interaction between $\mathrm{P}$ or $\mathrm{S}$ sites, located around $\mathrm{S}_{\mathrm{v}}$ position, and $\mathrm{O}$ atoms. In addition to the energy barrier issue for incapability of $\mathrm{O}_{2}$ evolution in our photocatalytic experiment, the presence of $\mathrm{S}_{\mathrm{v}}$ plays its own role in strongly adsorbing oxygen intermediate species thereby suppressing the generation of $\mathrm{O}_{2}$ gas (Supporting information).

The present photocatalysis offers a simpler and lower-cost approach to quantitatively extract pure $\mathrm{H}_{2}$ gas from neutral water in quantity. Even more attractively, the catalyst in this photocatalytic system avoids the complicated step to separate pure $\mathrm{H}_{2}$ gas from the explosive gas mixture, containing hydrogen and oxygen, directly achieved through the overall water splitting [3]. However, further investigation is indeed needed to unveil a clear picture of the surface chemistry and lattice structure of the atomic-layered $\mathrm{NiPS}_{3}$ crystals regarding the oxidation products. We believe that, with further research, the photocatalytic activity can be improved under sunlight, for instance, via separating the oxidation products from their surface or integrating $\mathrm{NiPS}_{3}$ nanosheets with suitable co-catalysts [47]. The construction of a highly active photocatalysts based on 2D ultrathin $\mathrm{NiPS}_{3}$ crystals paves a way to develop practical solar fuel applications and acquire new microscopic insights into photocatalytic water-splitting. Consequently, our discovery is expected to make a contribution to develop next generation solar-fuel catalysts for $\mathrm{H}_{2}$ production.

\section{Conclusions}

In summary, we, for the first time, grow ultrathin two-dimensional $\mathrm{NiPS}_{3}$ nanosheets through CVD method on different substrates. The thickness of the hexagonal $\mathrm{NiPS}_{3}$ nanosheets, whose lateral size is larger than $15 \mu \mathrm{m}$, is about few atomic layers $(\leq 3.5 \mathrm{~nm}$ ). Significantly, these $\mathrm{NiPS}_{3}$ nanosheets can be directly utilized as a novel photocatalyst to split water for hydrogen evolution under solar lights irradiation without any co-catalyst and sacrificial agents. The band structure of the 2D ultrathin $\mathrm{NiPS}_{3}$ was systematically studied via using ultraviolet photoelectron spectrometer and electrochemical impedance spectroscopy. The appropriate position of the band edge is crucial for photocatalytic water splitting. Our discovery opens a new avenue to develop next generation solar-fuel catalysts for $\mathrm{H}_{2}$ production.

\section{Acknowledgment}

This work was supported by Ministry of Science and Technology of China (No. 2016YFA0200700), National Natural Science Foundation of China (Nos. 61625401, 61574050 and 11674072), Strategic Priority Research Program of the Chinese Academy of Sciences (Grant No. 4), and CAS Key Laboratory of Nanosystem and Hierarchical Fabrication. The authors also gratefully acknowledge the support of Youth Innovation Promotion Association CAS.

\section{Appendix A. Supporting information}

Supplementary data associated with this article can be found in the online version at http://dx.doi.org/10.1016/j.nanoen.2017.09.017.

\section{References}

[1] A. Iwase, Y.H. Ng, Y. Ishiguro, A. Kudo, R. Amal, J. Am. Chem. Soc. 133 (2011) 11054-11057.

[2] X. Xue, W. Zang, P. Deng, Q. Wang, L. Xing, Y. Zhang, Z.L. Wang, Nano Energy 13 (2015) 414-422.

[3] B.A. Pinaud, J.D. Benck, L.C. Seitz, A.J. Forman, Z. Chen, T.G. Deutsch, B.D. James, K.N. Baum, G.N. Baum, S. Ardo, H. Wang, E. Miller, T.F. Jaramillo, Energy Environ. Sci. 6 (2013) 1983-2002.

[4] L. Liao, Q. Zhang, Z. Su, Z. Zhao, Y. Wang, Y. Li, X. Lu, D. Wei, G. Feng, Q. Yu, Nat. Nano 9 (2014) 69-73.

[5] K. Maeda, K. Teramura, K. Domen, J. Catal. 254 (2008) 198-204.

[6] J. Di, J. Xia, H. Li, Z. Liu, Nano Energy 35 (2017) 79-91.

[7] H. Li, Y. Shi, M.-H. Chiu, L.-J. Li, Nano Energy 18 (2015) 293-305.

[8] C. Tan, Z. Lai, H. Zhang, Adv. Mater. 29 (2017) 1701392.

[9] X. Zhang, Z. Lai, C. Tan, H. Zhang, Angew. Chem. Int. Ed. 55 (2016) 8816-8838.

[10] M. Zhou, X.W.D. Lou, Y. Xie, Nano Today 8 (2013) 598-618.

[11] C. Tan, X. Cao, X.-J. Wu, Q. He, J. Yang, X. Zhang, J. Chen, W. Zhao, S. Han, G.H. Nam, Chem. Rev. 117 (2017) 6225-6331.

[12] Q. Lu, Y. Yu, Q. Ma, B. Chen, H. Zhang, Adv. Mater. 28 (2016) 1917-1933.

[13] X. Wang, K. Maeda, A. Thomas, K. Takanabe, G. Xin, J.M. Carlsson, K. Domen, M. Antonietti, Nat. Mater. 8 (2009) 76-80.

[14] Y. Zheng, L. Lin, B. Wang, X. Wang, Angew. Chem. Int. Ed. 54 (2015) 12868-12884.

[15] J. Liu, Y. Liu, N. Liu, Y. Han, X. Zhang, H. Huang, Y. Lifshitz, S.-T. Lee, J. Zhong, Z. Kang, Science 347 (2015) 970-974.

[16] X. She, J. Wu, J. Zhong, H. Xu, Y. Yang, R. Vajtai, J. Lou, Y. Liu, D. Du, H. Li, P.M. Ajayan, Nano Energy 27 (2016) 138-146.

[17] J. Liu, Y. Zhang, L. Lu, G. Wu, W. Chen, Chem. Commun. 48 (2012) 8826-8828.

[18] Z. Li, C. Kong, G. Lu, J. Phys. Chem. C 120 (2015) 56-63.

[19] Z.-F. Huang, J. Song, X. Wang, L. Pan, K. Li, X. Zhang, L. Wang, J.-J. Zou, Nano Energy. http://dx.doi.org/10.1016/j.nanoen.2017.08.032.

[20] X. Li, X. Wu, J. Yang, J. Am. Chem. Soc. 136 (2014) 11065-11069.

[21] K.-z. Du, X.-z. Wang, Y. Liu, P. Hu, M.I.B. Utama, C.K. Gan, Q. Xiong, C. Kloc, ACS Nano 10 (2015) 1738-1743.

[22] X. Zhang, X. Zhao, D. Wu, Y. Jing, Z. Zhou, Adv. Sci. 3 (2016) 1600062.

[23] R. Nitsche, Le J. De Phys. Colloq. 36 (1975) (C3-9-C3-C15).

[24] R. Nitsche, P. Wild, Mater. Res. Bull. 5 (1970) 419-423.

[25] C.-T. Kuo, M. Neumann, K. Balamurugan, H.J. Park, S. Kang, H.W. Shiu, J.H. Kang, B.H. Hong, M. Han, T.W. Noh, Sci. Rep. 6 (2016) 20904.

[26] F. Wang, Y. Li, T.A. Shifa, K. Liu, F. Wang, Z. Wang, P. Xu, Q. Wang, J. He, A Angew. Chem. 128 (2016) 7033-7038.

[27] G. Kresse, J. Furthmüller, Phys. Rev. B 54 (1996) 11169-11186.

[28] P.E. Blöchl, Phys. Rev. B 50 (1994) 17953.

[29] J.P. Perdew, K. Burke, M. Ernzerhof, Phys. Rev. Lett. 77 (1996) 3865.

[30] X. Li, J. Dong, J.C. Idrobo, A.A. Puretzky, C.M. Rouleau, D.B. Geohegan, F. Ding, K. Xiao, J. Am. Chem. Soc. 139 (2017) 482-491.

[31] P. Kumar, B. Viswanath, Cryst. Growth Des. 16 (2016) 7145-7154.

[32] L. Zhang, K. Liu, A.B. Wong, J. Kim, X. Hong, C. Liu, T. Cao, S.G. Louie, F. Wang, P. Yang, Nano Lett. 14 (2014) 6418-6423.

[33] Y. Liu, M. Weinert, L. Li, Phys. Rev. Lett. 108 (2012) 115501.

[34] M. Bernasconi, G. Marra, G. Benedek, L. Miglio, M. Jouanne, C. Julien, M. Scagliotti, M. Balkanski, Phys. Rev. B 38 (1988) 12089.

[35] M. Piacentini, F. Khumalo, C. Olson, J. Anderegg, D. Lynch, Chem. Phys. 65 (1982) 289-304.

[36] M. Piacentini, F. Khumalo, G. Leveque, C. Olson, D. Lynch, Chem. Phys. 72 (1982) 61-71.

[37] D. Voiry, R. Fullon, J. Yang, C. de Carvalho Castro e Silva, R. Kappera, I. Bozkurt, D. Kaplan, M.J. Lagos, P.E. Batson, G. Gupta, A.D. Mohite, L. Dong, D. Er, V.B. Shenoy, T. Asefa, M. Chhowalla, Nat. Mater. 15 (2016) 1003-1009. 
[38] W. Zhou, X. Zou, S. Najmaei, Z. Liu, Y. Shi, J. Kong, J. Lou, P.M. Ajayan, B.I. Yakobson, J.-C. Idrobo, Nano Lett. 13 (2013) 2615-2622.

[39] J. Hong, Z. Hu, M. Probert, K. Li, D. Lv, X. Yang, L. Gu, N. Mao, Q. Feng, L. Xie, J. Zhang, D. Wu, Z. Zhang, C. Jin, W. Ji, X. Zhang, J. Yuan, Z. Zhang, Nat. Commun. 6 (2015) 6293.

[40] R. Brec, D. Schleich, G. Ouvrard, A. Louisy, J. Rouxel, Inorg. Chem. 18 (1979) 1814-1818.

[41] C.-T. Kuo, K. Balamurugan, H.W. Shiu, H.J. Park, S. Sinn, M. Neumann, M. Han, Y.J. Chang, C.-H. Chen, H.-D. Kim, Curr. Appl. Phys. 16 (2016) 404-408.

[42] A. Ishikawa, T. Takata, J.N. Kondo, M. Hara, H. Kobayashi, K. Domen, J. Am. Chem. Soc. 124 (2002) 13547-13553.

[43] B.A. Pinaud, Z. Chen, D.N. Abram, T.F. Jaramillo, J. Phys. Chem. C 115 (2011) $11830-11838$.

[44] T. Yao, R. Chen, J. Li, J. Han, W. Qin, H. Wang, J. Shi, F. Fan, C. Li, J. Am. Chem. Soc. 138 (2016) 13664-13672.

[45] A. Aruchamy, H. Berger, F. Lévy, J. Electrochem. Soc. 136 (1989) 2261-2265.

[46] Y. Matsumoto, J. Solid State Chem. 126 (1996) 227-234.

[47] J. Yang, H. Yan, X. Zong, F. Wen, M. Liu, C. Li, Philos. Trans. R. Soc. A: Math. Phys. Eng. Sci. 371 (2013) 20110430.

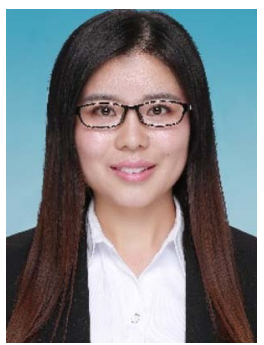

Fengmei Wang received her BS degree in Applied Chemistry from Hunan University in 2012. Then she obtained her Ph.D. degree from National Center for Nanoscience and Technology (NCNST), University of Chinese Academy of Sciences (UCAS). She joined National Center for Nanoscience and Technology (NCNST) in 2017, where she worked as an assistant professor. Her research interests include synthesis and development of transition metal chalcogenides for electrocatalytic and photocatalytic water splitting.

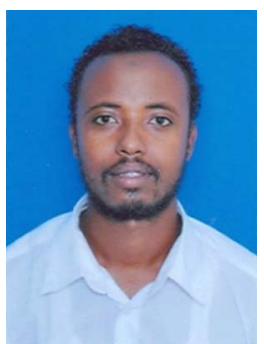

Tofik Ahmed Shifa received his B.Sc. degree in Applied Chemistry from Arbaminch University, Ethiopia in 2007. He then obtained his M.Sc. degree in Analytical Chemistry from Haramaya University, Ethiopia in 2011 and worked as lecturer in the Department of Chemistry. Currently, he is pursuing a Ph.D. degree under the supervision of Prof. Jun $\mathrm{He}$ at National Center for Nanoscience and Technology (NCNST), Beijing, China. His research focuses on controllably synthesizing two dimensional layered materials for catalysis of water splitting reactions.

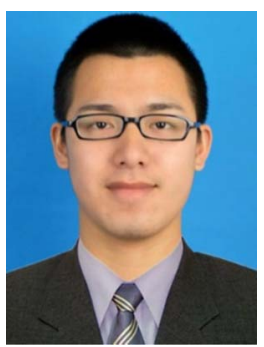

Peng He received his B.Sc. and M.Sc. degrees in Electronic Science and Technology from Beijing University of Chemical Technology (BUCT) in 2014 and 2017. He will be a Ph.D. candidate in National Center for Nanoscience and Technology (NCNST) on September 2017. His research mainly focuses on the first principle theory simulation and calculation based low-dimensional materials in optoelectronic devices, electrochemical catalytic and the Density Functional Theory (DFT) study.

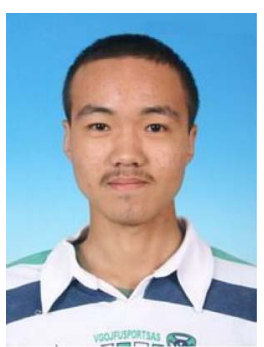

Zhongzhou Cheng received his B.Sc. degree in Materials Physics and Chemistry from University of Science and Technology Beijing in 2012. Currently, he is pursuing a $\mathrm{Ph}$.D. degree as a joint student under the supervision of Prof. Jun $\mathrm{He}$ at National Center for Nanoscience and Technology (NCNST) and Prof. Quanlin Liu at University of Science and Technology (USTB), Beijing, China. His research focuses on the synthesis and application of photocatalytic water splitting based on nanomaterials.

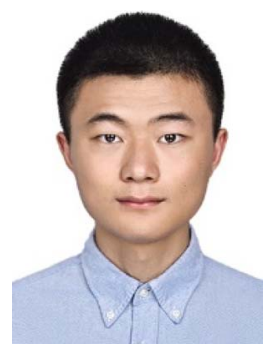

Junwei Chu received his B.S. degree in Electronic Science and Technology from University of Electronic Science and Technology of China (UESTC) in 2015. Currently, he is a $\mathrm{Ph} . \mathrm{D}$. candidate in Prof Jun He's group at National Cente for Nanoscience and Technology and Prof. Jie Xiong's group at UESTC. He is familiar with circuits design, optical path design and MATLAB programming. His scientific research concentrates on the Raman and dynamic process of lightmatter interaction in optoelectronic devices based on in trinsic 2D materials and 2D heterostructures.

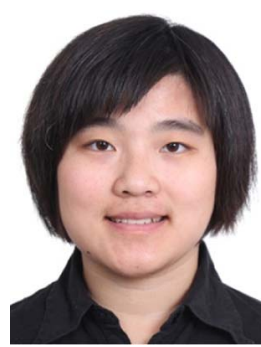

Yang Liu received her B.Sc. degree in Chemistry from Capital Normal University in 2016. Currently, she is striving for M.Sc. degree under the supervision of Prof. Jun $\mathrm{He}$ at National Center for Nanoscience and Technology (NCNST), Beijing, China. Her research interests cover the controllable synthesis of transition-metal chalcogenides for electrocatalytic water splitting.

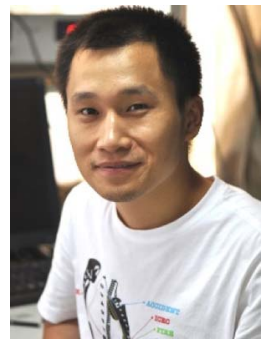

Zhenxing Wang received his B.S. and Ph.D. degrees from University of Science and Technology of China (USTC) in 2002 and 2009, respectively. After two-year postdoctoral work at Peking University, he joined National Center for Nanoscience and Technology (NCNST) in 2011, where currently he is a Professor. His current research interests focus on low dimensional materials and optoelectronic devices.

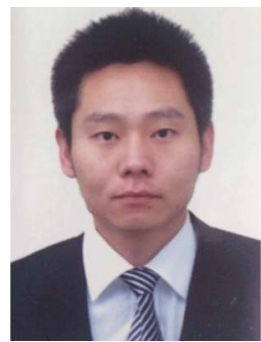

Feng Wang received his Bachelor's degree from the Department of Materials Physics, Yanshan University, in 2010. Then he received his Master's degree in engineering from Zhejiang University in 2013. After that, he worked as a Research Assistant in the Department of Mechanical and Automation Engineering, the Chinese University of Hong Kong. Now he is a Ph.D. candidate under the supervision of Prof. He in the National Center for Nanoscience and Technology (NCNST), Chinese Academy of Sciences. His current research is developing two-dimensional materials for electronic and optoelectronic applications.

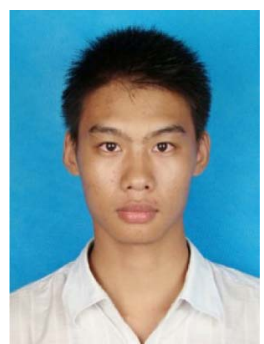

Yao Wen received his B.S. degree in Materials Physics from Hubei University in 2014. Now he is a Ph.D. candidate under the supervision of Prof. He and Prof. Jiang in the National Center for Nanoscience and Technology (NCNST). His current research focuses on the synthesis and devices of low dimensional semiconductor materials for electronic and optoelectronic applications. 


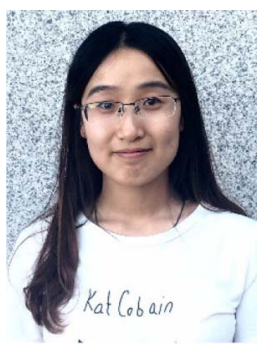

Lirong Liang received her B.Sc. degree in China University of Mining and Technology (Beijing) in 2014. Then, she joined the Analysis and Testing Center of Institute of Chemistry, Chinese Academy of Sciences in 2014. The specific position is instrument supervisor of the electron microscope group, responsible for the SEM and TEM related operations and measurements.

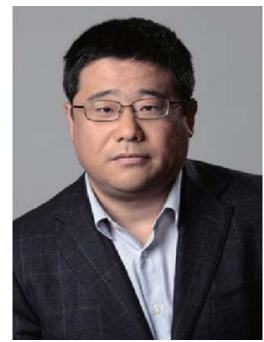

Jun He received his Ph.D. in Semiconductor Physics from the Institute of Semiconductors, Chinese Academy of Sciences (CAS), in 2003. Then he worked successively at Applied Physics Department of Technische Universiteit Eindhoven, Netherlands, Material Department of University of California, Santa Babara, and California NanoSystem Institute (CNSI), University of California, Los Angeles, USA He joined the "100-Talents" Program of CAS in Nov. 2010 and became a Full Professor of NCNST since then. Professor He's main research interest is the synthesis, characterization, and devices of low dimensional semiconductor materials. 